Enrique, Nicolás; D’ambrosio, Marianela; Speroni, Francisco

Trayectorias Universitarias, 6(10), e025, 2020

ISSN 2469-0090 | https://doi.org/10.24215/24690090e025

https://revistas.unlp.edu.ar/TrayectoriasUniversitarias

Universidad Nacional de La Plata

La Plata | Buenos Aires | Argentina

\title{
Enseñanza de Anatomía e Histología en tiempos de pandemia: adaptaciones para una cursada a distancia
}

\section{Teaching of Anatomy and Histology in times of pandemic: adaptations for a distance study}

Ibáñez Shimabukuro, Marina https://orcid.org/0000-0002-8612-3057 ibanez.marina@gmail.com

Cátedra de Anatomía e Histologia (comisión F.O.P.B.)

| Departamento de Ciencias Biológicas| Facultad de Ciencias Exactas | UNLP | Argentina

Gangoiti, María Virginia

https://orcid.org/0000-0001-8058-973X

virginiagangoiti@yahoo.com.ar

Cátedra de Anatomía e Histologia (comisión F.O.P.B.)

| Departamento de Ciencias Biológicas| Facultad de

Ciencias Exactas | UNLP | Argentina

D’ambrosio, Marianela

https://orcid.org/0000-0001-5099-0813

manedambrosio@gmail.com

Cátedra de Anatomía e Histologia (comisión F.O.P.B.)

| Departamento de Ciencias Biológicas| Facultad de

Ciencias Exactas | UNLP | Argentina
Sbaraglini, María Laura

https://orcid.org/0000-0002-4671-2658

mariasbara@gmail.com

Cátedra de Anatomía e Histologia (comisión F.O.P.B.)

| Departamento de Ciencias Biológicas| Facultad de

Ciencias Exactas | UNLP | Argentina

Enrique, Nicolás

https://orcid.org/0000-0001-9130-6678

nicoenrique@gmail.com

Cátedra de Anatomía e Histologia (comisión F.O.P.B.)

| Departamento de Ciencias Biológicas| Facultad de

Ciencias Exactas | UNLP | Argentina

Speroni, Francisco

https://orcid.org/0000-0001-5787-8111

franciscosperoni@gmail.com

Cátedra de Anatomía e Histologia (comisión F.O.P.B.)

| Departamento de Ciencias Biológicas| Facultad de

Ciencias Exactas | UNLP |Argentina

\section{RESUMEN}

La pandemia por el coronavirus impuso una situación sanitaria extraordinaria cuyas medidas de contención y prevención gubernamentales implicaron el aislamiento social y consecuentemente por disposición de la UNLP, la interrupción de clases presenciales a partir del 16 de marzo del 2020.

En función de las nuevas circunstancias, docentes y estudiantes atravesamos desafíos que exaltan el carácter complejo y dinámico de la trama educativa y que revelan de manera patente esa amalgama de lo académico, lo político, lo institucional, lo cultural y lo histórico como invita a pensar el eje temático elegido para este relato de experiencia pedagógica.

Contemplar el impacto del aislamiento social en las realidades de lxs estudiantes y docentes nos impulsó a repensar objetivos y horizontes. Y volver sobre nuestras prácticas nos llevó a proponer una reorganización curricular, rediseñar materiales didácticos (cuestionarios, videos de clases) y adaptar los dispositivos de evaluación.

Compartimos aquí los desafíos pedagógicos sobre los cuales reflexionamos, en tanto profesionales de la educación, y las experiencias de transformación que estamos desarrollando en la comisión para Farmacia, Licenciatura en Óptica Ocular y Optometría, Profesorado en Física, Profesorado en Química y Licenciatura en Biotecnología y Biología Molecular de la Cátedra de Anatomía e Histología (AeH com. FOBP) de la Facultad de Ciencias Exactas, UNLP.

PALABRAS CLAVE

Anatomía e Histología, Pandemia,

Cursada a Distancia 


\section{KEY WORDS}

Anatomy and Histology, Pandemic,

Studied at a Distance

\section{ABSTRACT}

The coronavirus pandemic imposed an extraordinary health situation whose government containment and prevention measures implied social isolation and consequently, by order of the UNLP, the interruption of face-to-face classes as of March 16, 2020.

Depending on the new circumstances, teachers and students go through challenges that exalt the complex and dynamic nature of the educational fabric and that clearly reveal that amalgam of the academic, political, institutional, cultural and historical aspects as it invites us to think. the thematic axis chosen for this narrative of pedagogical experience.

Contemplating the impact of social isolation on the realities of students and teachers prompted us to rethink goals and horizons. And going back to our practices led us to propose a curricular reorganization, redesign teaching materials (questionnaires, class videos) and adapt the assessment devices.

We share here the pedagogical challenges on which we reflect, as education professionals, and the transformation experiences that we are developing in the commission for Pharmacy, Bachelor of Ocular Optics and Optometry, Faculty of Physics, Faculty of Chemistry and Bachelor of Biotechnology and Molecular Biology of the Chair of Anatomy and Histology (AeH com. FOBP) of the Faculty of Exact Sciences, UNLP. 


\section{PRESENTACIÓN}

\section{La asignatura y el contexto académico}

Anatomía e Histología (AeH) es esencial para aquellas carreras relacionadas con la salud y complementa la formación en ciencias en otros planes de estudio. Los saberes de AeH son necesarios para aprender Fisiología, Fisiopatología, Farmacología, y cualquier otra materia en la que el cuerpo humano es el objeto de estudio. En la Facultad de Ciencias Exactas de la UNLP, AeH se brinda de manera cuatrimestral y en el caso de nuestra comisión se incluye de manera obligatoria entre el segundo o tercer año de los planes de estudio de las carreras de Farmacia, Licenciatura en Óptica Ocular y Optometría, Profesorado en Física, Profesorado en Química; y de manera optativa para la carrera de Licenciatura en Biotecnología y Biología Molecular.

El paso por AeH pretende generar en lxs estudiantes una imagen precisa y completa de la organización morfológica del cuerpo humano vivo, orientada hacia la relación estructura-función de los sistemas y aparatos. Persigue además, articular con los conocimientos desarrollados en materias anteriores, así como también consolidar el andamiaje conceptual para asignaturas posteriores.

En la cursada correspondiente al primer cuatrimestre del 2020 consideramos una matrícula de 45 estudiantes con Ixs que llegamos a compartir 6 encuentros presenciales durante las dos primeras semanas de marzo (para cada subcomisión se pautan 3 encuentros semanales: una Teoría, un Seminario y un Trabajo Práctico).

\section{Antecedentes sobre plataformas digitales: la Cátedra Virtual como espacio de trabajo consolidado}

La cátedra de AeH cuenta con un espacio en línea soportado en la plataforma educativa Moodle de la Facultad de Ciencias Exactas, UNLP que denominamos Cátedra Virtual. Desde hace más de 10 años la Cátedra Virtual de AeH funciona como ampliación del aula del curso presencial; proporciona un canal de asidua comunicación con una intencionalidad de aprendizaje continuo y dialógico, cuya función tras- 
ciende la de mero repositorio de documentos. En efecto, la Cátedra Virtual comenzó a ser el escenario de diversas actividades (foros de discusión y simulacros de exámenes, entre otros) que con el correr del tiempo fueron reemplazadas, otras mejoradas o sostenidas en sintonía con la constante acomodación a los desafíos que fuimos percibiendo, pero que en definitiva fueron consolidando un espacio de labor intensiva con gran participación por parte de lxs estudiantes (Felice y cols., 2013; Felice y cols., 2015; Ibáñez Shimabukuro y cols., 2015; Ibáñez Shimabukuro y cols., 2016; Ibáñez y Speroni, 2019).

\section{Desafíos pedagógicos en torno a la condición de aislamiento so- cial por el coronavirus y a la consecuente migración de la enseñan- za a entornos virtuales.}

La condición de distanciamiento social, la interrupción de nuestra cotidianeidad y las preocupaciones derivadas de la pandemia componen un escenario complejo que afecta de manera particular a cada sujeto. Sumado esto a la suspensión de clases presenciales da por resultado importantes cambios en la vida estudiantil que pueden reflejarse en la desorganización de las rutinas y la postergación del estudio.

La condición de distanciamiento social, la interrupción de nuestra cotidianeidad y las preocupaciones derivadas de la pandemia componen un escenario complejo que afecta de manera particular a cada sujeto. Sumado esto a la suspensión de clases presenciales da por resultado importantes cambios en la vida estudiantil que pueden reflejarse en la desorganización de las rutinas y la postergación del estudio.

Por otra parte, el desplazamiento desde el formato presencial al de Educación a Distancia demanda un mayor grado de autonomía por parte de Ixs estudiantes. Si bien en $\mathrm{AeH}$ ya veníamos utilizando a la Cátedra Virtual de manera intensa como extensión del aula presencial, ahora el territorio virtual cobra centralidad y adaptarse a estas nuevas formas de contactarnos requiere un esfuerzo adicional. En este sentido tal migración supone un desafío también para lxs docentes que debemos aprender nuevas herramientas y soportes.

\section{DESCRIPCIÓN DE LA EXPERIENCIA}

A partir de la suspensión de las clases presenciales realizamos reuniones de cátedra periódicas por videoconferencia con la totalidad del plantel ( 1 ayudante alumna, 2 ayudantes diplamdxs, 2 jefas de TP y 1 profesor). Allí fuimos discutiendo y decidiendo las distintas acciones en cuanto al régimen de cursada que tomaron un matiz flexible. Asumir una condición de provisionalidad nos permitió adaptarnos al 
dinamismo de las pautas institucionales e ir explorando y ajustando actividades y metodologías que describimos a continuación:

\section{Tutoría - acompañamiento docente}

Una de las preocupaciones iniciales fue conocer las realidades singulares de nuestrxs estudiantes. Por un lado, planteamos la necesidad de estrechar el contacto, contemplar situaciones extraordinarias o que requirieran alguna intervención desde nuestro rol docente. Por otro, dada la transición de la enseñanza a espacios netamente virtuales, relevar la posibilidad de uso de computadoras y la accesibilidad a internet fue fundamental a fin de no profundizar condiciones de desigualdad.

Nos propusimos dividirnos de modo tal que cada estudiante tuviera asignado un/a docente para un seguimiento más personalizado. En una primera comunicación individual por correo electrónico (proveniente de la información de contacto del lxs usuarixs del Moodle) incluimos el siguiente texto con una serie de preguntas que elaboraron y compartieron Ixs docentes de la cátedra de Fisiología perteneciente a la misma área de AeH, dentro del Departamento de Ciencias Biológicas de la Facultad:

Hola -----! Soy -------, docente de la cátedra de Anatomía e Histología.

Me comunico con vos para saber cómo te encontrás y para que me tengas de contacto como docente de la cátedra.

Mientras dure la cuarentena, vamos a hacer lo posible para trabajar algunos aspectos del curso en forma virtual.

Te pido que nos ayudes con la siguiente información para que podamos saber qué estrategias podemos implementar:

1) ¿Estás cursando la materia?

2) ¿Estás recibiendo los mails que notifican de los posteos en foros, etc?

3) ¿Tenés acceso a internet como para seguir las actividades virtuales?

4) ¿Tenés acceso a una computadora para realizar trabajos en un archivo de Word?

5) ¿Sabés si hay compañerxs que no tengan acceso a internet y eso les impida conectarse y seguir las actividades? En caso afirmativo, ¿conocés alguna otra manera mediante la cual nos podríamos comunicar con algunx de ellxs?

6) ¿Hay alguna sugerencia / pedido que se te ocurra para mejorar la interacción virtual con la cátedra?

Como información te comento que Movistar y Personal liberaron el consumo de datos para los dominios que sean de unlp.edu.ar por lo que cualquier persona que tenga esos proveedores puede ingresar a Cátedras Virtuales sin tener datos móviles disponibles o conexión a internet.

Cualquier consulta o inquietud, no dudes en escribirme!

Por favor te pido me confirmes recepción de este correo electrónico.

Saludos, 
Luego de este primer contacto, para el cual tuvimos una respuesta casi total, volvimos a utilizar este canal para consultas individuales y para la organización de la evaluación online. Asimismo, la figura de tutor/a se mantuvo como referente de contacto, acompañamiento y corrección de actividades a fin de facilitar la comunicación y promover adherencia de Ixs estudiantes a la cursada.

\section{Videos explicativos}

A los videos que desde el año pasado resumían ciertos temas puntuales incorporamos videos semanales a modo de sustituto de las clases teóricas de la cursada presencial. En una etapa posterior también incorporamos videos explicativos de los contenidos habitualmente desarrollados en los Trabajos Prácticos (estudio y observación de preparados histológicos empleando fotos de los preparados de la cátedra).

Respecto a esta modalidad asincrónica de transmisión Ixs estudiantes rescataron varias ventajas; entre ellas: poder mirar los videos en cualquier momento, pausar para tomar apuntes, repetir las partes que fueran necesarias. De nuestra parte les aconsejamos que previo a ver los videos, leyeran el cuestionario correspondiente al tema de forma tal de escuchar con un interés focalizado sobre preguntas centrales y con ese mismo propósito también se publicaron en la Cátedra Virtual preguntas específicas para "seguir la Teoría".

Respecto a esta modalidad asincrónica de transmisión Ixs estudiantes rescataron varias ventajas; entre ellas: poder mirar los videos en cualquier momento, pausar para tomar apuntes, repetir las partes que fueran necesarias. De nuestra parte les aconsejamos que previo a ver los videos, leyeran el cuestionario correspondiente al tema de forma tal de escuchar con un interés focalizado sobre preguntas centrales y con ese mismo propósito también se publicaron en la Cátedra Virtual preguntas específicas para "seguir la Teoría".

\section{Tareas semanales para entregar}

Estas actividades se propusieron como de participación obligatoria. Procuramos que las tareas no fueran muy complejas, ni muy extensas, pero que representaran un buen estímulo para estudiar los temas que se van viendo semanalmente. Si bien estipulamos una fecha de entrega para tener el presente (virtual) de la semana, contemplamos excepciones. Para organizar estas actividades utilizamos el módulo de tareas que ofrece el Moodle que les permite a Ixs estudiantes subir documentos y a Ixs docentes, realizar devoluciones. 


\section{Consultas por foros y encuentros sincrónicos}

Mantener la interacción entre docentes y estudiantes y también entre pares es fundamental y quizás sea uno de los aspectos más desafiantes en la educación a distancia. Al respecto, dentro de los recursos que favorecen la interacción en la Cátedra Virtual veníamos utilizando los Foros que permiten intercambiar dudas, experiencias, etc., de manera asincrónica y ordenada por tema.

Por otro lado, en cuanto a comunicación sincrónica, experimentamos con diversos formatos. En una primera instancia trabajamos con las salas de consulta dentro del Moodle que tienen la ventaja -respecto a los Foros- del diálogo en tiempo real. Sin embargo, el formato de chat nos resultó poco dinámico y limitado (por ejemplo, no es posible compartir imágenes, algo fundamental para la enseñanza de AeH). En consecuencia, con el correr de las semanas fuimos reemplazando las salas de chat por encuentros sincrónicos utilizando plataformas privadas (Zoom y más adelante también Big Blue Button, esta última incorporada dentro del Moodle). Los encuentros (de asistencia voluntaria) por estos canales tuvieron más convocatoria con un máximo de 23 estudiantes simultáneos y pudieron en la mayoría de las ocasiones grabarse para su posterior consulta. En cuanto al nivel de interacción consideramos que este tipo de actividades es muy valiosa. Habitar un espacio de simultaneidad, en donde nos escuchamos y "nos vemos las caras", aporta al vínculo pedagógico al menos parte de lo que hemos perdido al abandonar las aulas como estructura comunicativa. No obstante, notamos algunas limitaciones para conversar y predomina la transmisión más bien unidireccional.

Habitar un espacio de simultaneidad, en donde nos escuchamos y "nos vemos las caras", aporta al vínculo pedagógico al menos parte de lo que hemos perdido al abandonar las aulas como estructura comunicativa. No obstante, notamos algunas limitaciones para conversar y predomina la transmisión más bien unidireccional.

\section{Adecuación y/o elaboración de material didáctico}

La virtualización de la educación supuso adecuar y/o generar material didáctico que pudiera suplir aquello que acontecía en las clases presenciales, y en ese sentido la explicitación de las intenciones pedagógicas cobró relevancia. En las primeras semanas de cuarentena acompañamos los cuestionarios que veníamos utilizando de manera corriente con un archivo de PowerPoint que operaba de guía-ayuda. Allí les brindábamos aclaraciones sobre a qué apuntaba cada pregunta 
y referencias a videos y material bibliográfico que en otras circunstancias se hubieran visto en la actividad presencial de Seminario. Con el correr de las semanas se pasó a directamente reformular los cuestionarios para incorporar secciones como la de "Objetivos", imágenes e interrogantes que orientaran e integraran los conceptos abordados en la semana y la reorganización de las preguntas que permitiera un trabajo más autónomo por parte de Ixs estudiantes. Dicha reformulación involucró en la mayoría de los casos aumentar el número de preguntas o ejercicios a fin de hacerlas más claros y explícitos.

Por otra parte, para las semanas en que se trataron los Sistemas Nervioso y Endócrino (temas de particular complejidad) fuimos incorporando dentro de la Cátedra Virtual algunos ejercicios con imágenes y de retroalimentación automática pensados para afianzar los conceptos y la terminología luego de haber visto los videos de Teoría correspondientes (Ilustraciones 1 y 2 ).

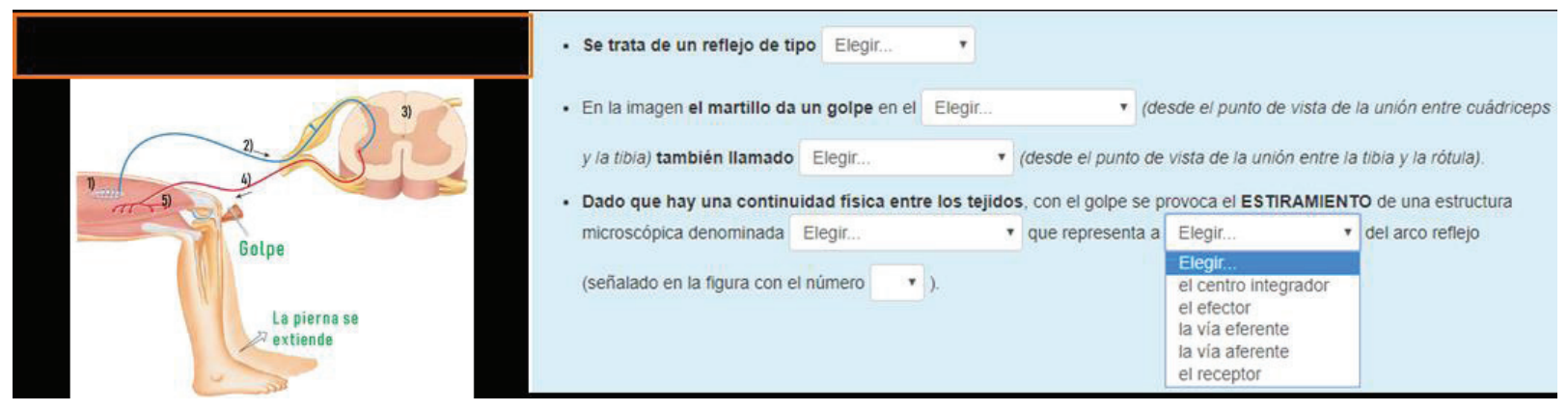

Ilustración 1 | (ejercicio de retroalimentación automática en la Cátedra Virtual-sistema nervioso)

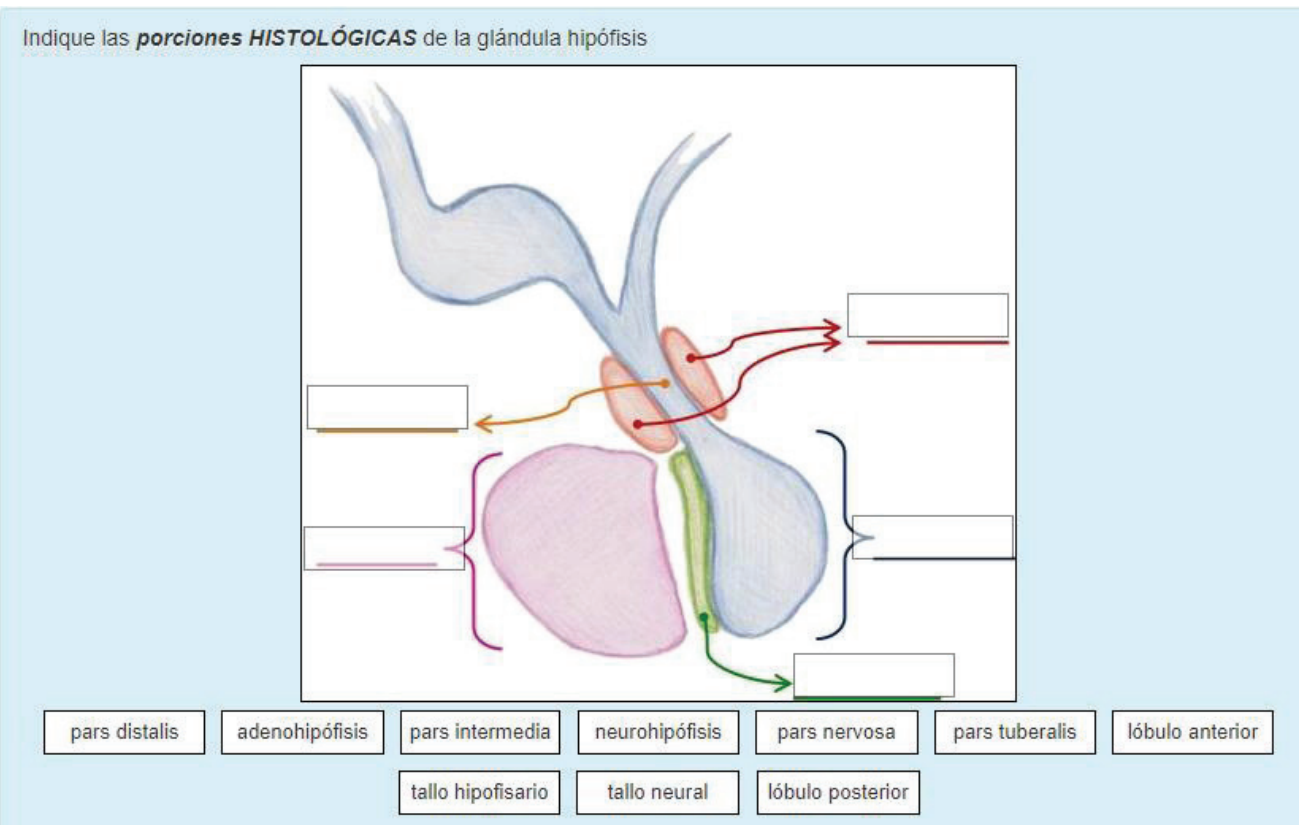

Ilustración 2 | (ejercicio de retroalimentación automática en la Cátedra Virtual-sistema endócrino) 


\section{Comunicación a Ixs estudiantes sobre las novedades en la orga- nización de la materia}

Reconocer el desasosiego que puede generar en Ixs estudiantes la interrupción abrupta de las clases presenciales y el carácter variable de los acontecimientos en el ámbito de la Universidad nos interpeló respecto a la responsabilidad de llevarles tranquilidad y brindar un marco de organización para informar los sucesivos cambios que fuimos implementando en la cursada. Mediante una infografía recopilamos de manera visual varios de los cambios introducidos en la cursada al comienzo de la cuarentena y explicitamos que los mantendríamos al tanto de las cuestiones que aún no estuvieran definidas (llustración 3). Luego a través de anuncios en los Foros de novedades de la Cátedra Virtual les comunicamos con el mayor tiempo posible de anticipación cómo resolveríamos cuestiones sensibles como la evaluación. Bajo estas acciones subyace un principio ético de reconocer a nuestrxs estudiantes y hacerles conocer que nos importan sus inquietudes.

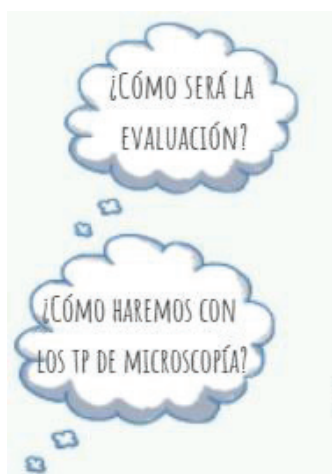

Les mantendremos informados sobre lo que se vaya resolviendo. Mientras tanto les aconsejamos que hagan lo posible para sostener el buen trabajo que venimos realizando y sacar lo mejor de la cursada por los canales virtuales. Por último, nos sumamos a la recomendación general de

\section{\#QuedáteEnCasa}

Ilustración 3 | (sección de infografía en la Cátedra Virtual sobre cambios en la cursada durante la cuarentena)

\section{Evaluación}

Consideramos que"la enseñanza, el aprendizaje y la evaluación deben constituirse en una unidad indisoluble" (Coll y cols., 2001). En palabras de Camilloni (s/f), "evaluar consiste, en principio, en emitir juicios de valor acerca de algo: objetos, conductas, planes. Estos juiciostienen una finalidad. La evaluación no tiene un fin en sí misma. No se evalúa por evaluar. Se evalúa para tomar decisiones con respecto a la marcha de un 
proceso". La evaluación trasciende las actividades de calificar y certificar. Consiguientemente, el diseño de la evaluación -más allá de los canales que se elijan- debe tener como norte los objetivos previamente discutidos y sopesados hacia el interior de cada plantel docente.

decidimos partir la materia en tres tramos (en vez de los dos habituales), cada uno con su parcial. Esta decisión se basó en que en un momento empezamos a notar que Ixs estudiantes no se estaban conectando tanto como al principio y que estaban demorando en entregar los cuestionarios semanales que envían por Moodle. Entonces, en vez de comenzar una nueva unidad que se desarrolla en tres semanas, nos pareció mejor hacer una pausa para integrar y estudiar lo visto en las primeras 5 semanas de clase (de las cuales 2 habíamos llegado a tener en forma presencial).

En nuestro caso particular, decidimos partir la materia en tres tramos (en vez de los dos habituales), cada uno con su parcial. Esta decisión se basó en que en un momento empezamos a notar que Ixs estudiantes no se estaban conectando tanto como al principio y que estaban demorando en entregar los cuestionarios semanales que envían por Moodle. Entonces, en vez de comenzar una nueva unidad que se desarrolla en tres semanas, nos pareció mejor hacer una pausa para integrar y estudiar lo visto en las primeras 5 semanas de clase (de las cuales 2 habíamos llegado a tener en forma presencial). A lxs estudiantes se les explicó el por qué de esta decisión, de forma de blanquear también frente a ellxs nuestras percepciones acerca del desarrollo del curso y de su participación.

Para el parcial elegimos un formato de opciones múltiples. Se definieron seis temas y se generaron cerca de 70 preguntas, de las cuales a cada estudiante le tocaron 4 de cada tema. Es decir 24 preguntas en total que el sistema asigna al azar a cada estudiante. A su vez, en cada una de esas 70 preguntas las opciones se presentan en distintos órdenes, por los cuales la probabilidad de tener dos parciales iguales es muy baja.

Durante el parcial, que duró 24 minutos, se invisibilizaron las demás actividades y recursos del curso virtual. Rindieron 38 estudiantes (de 45 en total).

El segundo parcial se desarrollará con un formato similar y el tercero será, si es posible, presencial en agosto de este año. Competencias como la redacción en la disciplina se trabajan y evalúan a través de entregas semanales de actividades como cuestionarios y descripciones histológicas.

Una vez terminado el parcial, cada estudiante pudo ver su nota y las respuestas correctas y contrastarlas con las elegidas.

Una encuesta post parcial es una fuente de información útil para ve- 
rificar si son necesarios ajustes en el diseño para futuras ocasiones de evaluación. En ese sentido, utilizamos los formularios de Google Forms para relevar la opinión de Ixs estudiantes.

A partir de las encuestas de participación voluntaria evidenciamos una gran aceptación al formato de evaluación (Ilustración 4) Valoraron positivamente la congruencia entre los contenidos enseñados y los evaluados; entre la dificultad y el tiempo de resolución; y en el formato de las preguntas. Respecto a los aspectos positivos, muchas personas destacaron la corta duración del examen, las preguntas concretas y sin "trampa" y la organización. Varias personas resaltaron que había sido valioso hacer este "parate" para repasar, estudiar y ver si se habían entendido o no los temas hasta ese momento. Frases como "Me senté estudiar y comprendí mejor los conceptos" o "Hacer el examen a esta altura de la cursada, sirvió muchísimo para hacer una pausa y repasar y entender todo lo que habíamos visto" avalan el sentido de regulación que propusimos como uno de los objetivos de este primer parcial.

En los aspectos negativos y sugerencias algunxs estudiantes mencionaron que el factor de tiempo acotado Ixs puso nerviosxs, relacionándolo en varios casos a no estar habituadxs al formato propuesto. En este sentido, también hubo quienes manifestaron que el formato de multiple choice les resultaba confuso; y por último algunas personas sugirieron que el parcial tuviese más preguntas.

Frases como "Me senté estudiar y comprendí mejor los conceptos" o "Hacer el examen a esta altura de la cursada, sirvió muchísimo para hacer una pausa y repasar y entender todo lo que habíamos visto" avalan el sentido de regulación que propusimos como uno de los objetivos de este primer parcial.

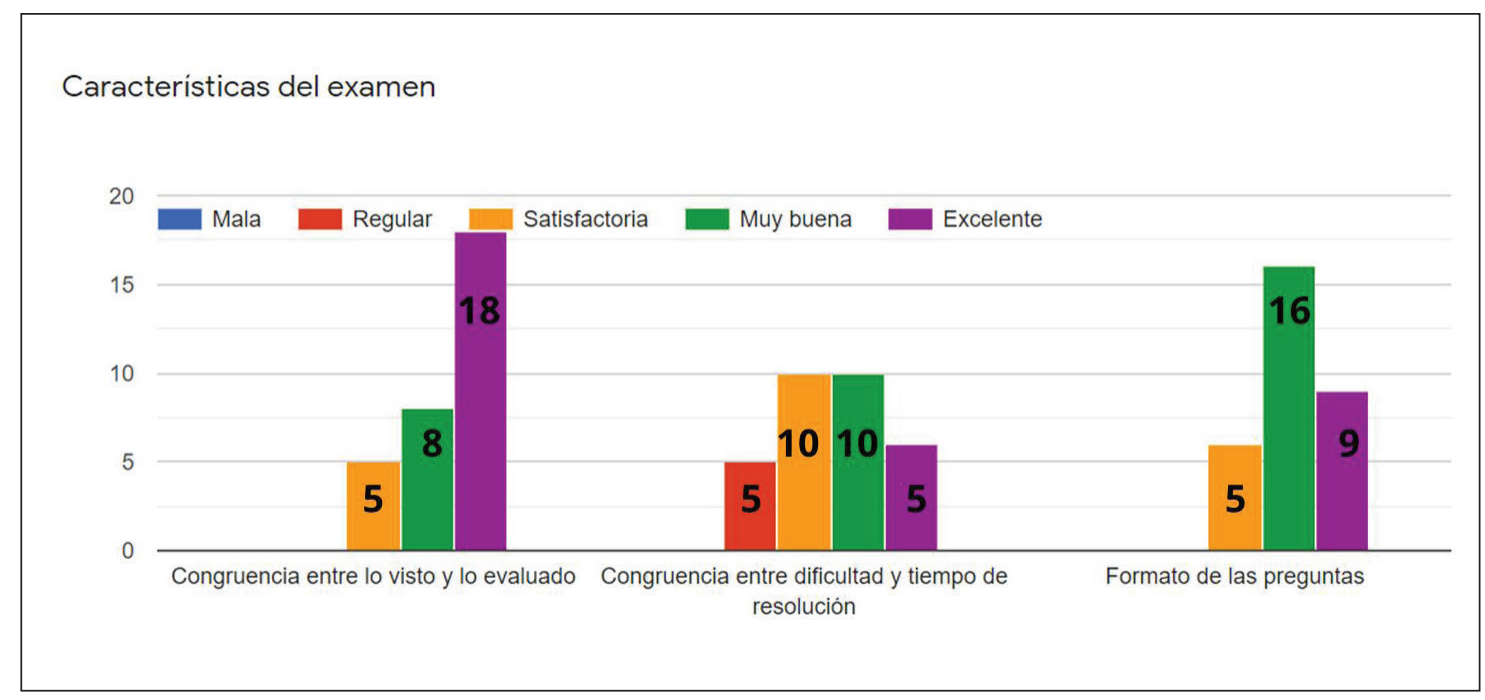

Ilustración 4 | (Fragmento de la encuesta post examen virtual / 31 encuestas sobre 38 personas que rindieron). 


\section{Preocupaciones sobre la validez de los exámenes virtuales}

Una inquietud general entre Ixs docentes ligada a la condición de virtualidad es cómo asegurar que Ixs estudiantes realicen la evaluación de manera individual, acudiendo exclusivamente a los conocimientos adquiridos sin consultar material bibliográfico, internet, etc.

Si bien encontramos que existen algunas herramientas informáticas que dificultan acciones fraudulentas -como por ejemplo bloquear el dispositivo mientras se esté completando el cuestionario-, nos pareció mejor recurrir a otro enfoque.

\section{En particular nosotrxs optamos por:}

- Diseñar un cuestionario en base a subconjuntos de preguntas aleatorizadas. De esta manera a partir de un banco de preguntas numeroso, cada estudiante recibe una combinación de preguntas única. Asimismo, en las preguntas de formato "Multiple choice" las opciones de respuestas presentan un ordenamiento al azar, lo que también obstaculiza el potencial intercambio de respuestas entre estudiantes.

- Acotar el tiempo de resolución del cuestionario. Por el tipo de preguntas que elegimos -que no incluye preguntas de desarrollo, ni procesos de razonamientos prolongados-, pudimos establecer un margen de tiempo bastante acotado que por un lado asegure su resolución total -si se tiene mediana certeza sobre las respuestas-, pero al mismo tiempo, exiguo como para disuadir cualquier intención de buscar respuestas en otros sitios. Para tener una buena aproximación de este tiempo pedimos a docentes externos, además de Ixs docentes de la cátedra, para realizar un simulacro de resolución y calculando un suplemento extrapolado para la situación de Ixs estudiantes.

- Explicitar las normas del examen a Ixs estudiantes y hacerles partícipes del acuerdo para que la evaluación sea válida. Publicamos las reglas del examen explicitando que debía resolverse individualmente y recurriendo exclusivamente a los conocimientos adquiridos. Además, explicamos las razones subyacentes a ciertas características del examen como por ejemplo por qué el tiempo de resolución sería limitado. Asimismo, elaboramos un examen simulado con preguntas no relacionadas a la asignatura a fin de que Ixs estudiantes pudieran familiarizarse anticipadamente con el mecanismo de interacción en el futuro cuestionario y con los formatos de preguntas. Todas estas acciones que bregan por la transparencia favorecen a que la instancia de evaluación sea legitimada por Ixs estudiantes. Luego se les solicitó como requisito para recibir la contraseña de acceso que enviaran individualmente un correo donde declarasen la aceptación de las normas y el compromiso de realizar el examen en solitario y sin recurrir a ayudas. 


\section{CONCLUSIONES}

En este contexto inédito de pandemia, que tiene a la incertidumbre como denominador común, problematizamos el valor de la continuidad de la cursada en dimensiones que exceden al contenido y que reivindican los aspectos metodológicos y la faceta vincular en la docencia.

Desde lo metodológico, del abanico presentado surge que algunos recursos y/o actividades son más útiles que otros y utilizados en mayor o menor medida por distintos grupos de estudiantes. En tal sentido, consideramos que la combinación de soportes y medios es una buena alternativa para atender la heterogeneidad de la clase. Por otra parte, la experiencia de más de 10 años usando las cátedras virtuales en sus diferentes formatos nos encontró con fortalezas y recursos ya preparados que se aplicaron en este cuatrimestre.

Desde lo metodológico, del abanico presentado surge que algunos recursos y/o actividades son más útiles que otros y utilizados en mayor o menor medida por distintos grupos de estudiantes. En tal sentido, consideramos que la combinación de soportes y medios es una buena alternativa para atender la heterogeneidad de la clase. Por otra parte, la experiencia de más de 10 años usando las cátedras virtuales en sus diferentes formatos nos encontró con fortalezas y recursos ya preparados que se aplicaron en este cuatrimestre.

En cuanto a los interrogantes en los que continuamos trabajando señalamos en las actividades que implican simultaneidad, ¿cómo hacer para que los encuentros por videosesiones sean más participativos? ¿qué actividades podrían incluirse? Por ejemplo, podríamos intercalar preguntas durante el desarrollo de alguna explicación para que Ixs estudiantes contesten por medio de una encuesta, aprovechando que algunas plataformas ofrecen esta herramienta accesoria. De esta manera podríamos obtener un poco más de información para la autorregulación de la clase si algún concepto hubiera ofrecido dificultades. Desafíos como éste vuelven más evidentes los aspectos multisensoriales que de manera más inadvertida y solapada suceden en una clase presencial y cuya ausencia podrían obstaculizar la enseñanza por los canales virtuales.

Siguiendo con los desafíos, como docentes tuvimos que reestructurar maneras de enseñar, atrevernos a comunicar por nuevos canales y sortear prejuicios al momento de considerar nuevos dispositivos de evaluación. Asimismo, el mayor tiempo que demanda la planificación de todo lo antedicho en conjunto con el hecho de suceder en un contexto de aislamiento - donde lo académico se confunde con lo doméstico- reviste una gran exigencia para lxs docentes. 
Por último, en relación a la dimensión vincular de la docencia, tal como propone la pedagoga argentina, Inés Dussel en el reciente conversatorio de "La clase en pantuflas": los extraños momentos que nos tocan vivir pueden ofrecer una "oportunidad para pensar profundamente a las clases" (Dussel, 2020). Así, nos propone ponderar las clases como el tiempo-espacio de suspenso donde pausamos por un momento el frenesí de noticias; la clase como un descanso en un lugar no mediado por la enfermedad y el miedo. Un lugar donde sucedan procesos de construcción colectiva, donde se tejan redes de conocimiento, pero también redes que desde lo pedagógico implican un valor afectivo. 


\section{BIBLIOGRAFIA}

Camilloni, A. R. (s.f.). “Las funciones de la evaluación. PFDC - Curso en Docencia Universitaria". Módulo 4: Programas de Enseñanza y Evaluación de aprendizajes. Facultad de Psicología - UBA. Disponible en: http://23118.psi. uba.ar/academica/cursos_actualizacion/recursos/funcioncamillioni.pdf

Coll, C., Martín, E. y Onrubia, J. (2001) (como se cita en Esquivel, J. M. (2009). "Evaluación de los aprendizajes en el aula: una conceptualización renovada". En: Martín, E. y Martínez, F.: Avances y desafíos en la evaluación educativa. Madrid: OEl y Fundación Santillana p. 127.

Dussel, I. [Canal ISEP]. (2020, abril 18). “La clase en pantuflas". Conversatorio virtual con Inés

Dussel | ISEP [Archivo de video]. Recuperado de https://youtu.be/6xKvCtBC3Vs

Felice, J. I., Sbaraglini, M. L., Speroni, F. (2013). “Entornos virtuales en procesos de enseñanza - Aprendizaje de la asignatura Anatomía e Histología". Actas II Simposio de Enseñanza de la Biología. Facultad de Humanidades y Ciencias de la Educación. Universidad Nacional de La Plata. Disponible en: http://simposiosceyn.fahce.unlp.edu.ar/biologia/Actas/Felice_et_al.pdf

Felice, J. I., Ibáñez Shimabukuro, M., Sbaraglini, M. L., Speroni, F. (2015). “Alcances y desafíos en la implementación de una Cátedra Virtual en la enseñanza y aprendizaje de Anatomía e Histología". III Jornadas de TIC e Innovación en el Aula UNLP."Enlaces entre Educación, Conocimiento Libre y Tecnología Digitales". Disponible en: http://sedici.unlp.edu. ar/handle/10915/48 788

Ibáñez, M., Sbaraglini, M. L., Speroni, F. J., Felice, J. I. (2015). “Cátedra Virtual en la enseñanza y aprendizaje de Anatomía e Histología. Estrategias para promover el trabajo colaborativo y la apropiación de vocabulario". En S. Provenzano (Presidente), Libro de resúmenes $1^{\circ}$ Congreso Franco-Argentino de Anatomía. Inauguración de la Cátedra Unesco UNI-TWIN de Anatomía Digital (UBA- Univ. Paris Descartes). $1^{\circ}$ Congreso Internacional Las TIC para la enseñanza e investigación en Anatomía (pp. 15-16). Buenos Aires: Facultad de Medicina de la Universidad de Buenos Aires, Facultad de Farmacia y Bioquímica y del Centro de Innovación en Tecnología y Pedagogía de la UBA.

Ibáñez Shimabukuro, M., Felice, J. I., Sbaraglini, M. L., Chuguransky, S., Gangoiti, M. V., Speroni, F. (2016). “Capítulo 5. Estrategias para el aprendizaje de la asignatura Anatomía e Histología en la FCE de la UNLP". En M. Insaurralde (Comp), Enseñar en las universidades y en los institutos de formación docente (pp. 55-64). Buenos Aires: Noveduc.

Ibáñez Shimabukuro, M. y Speroni, F. (2019). “Entornos Virtuales de Enseñanza y Aprendizaje en Anatomía e Histología". En: Libro de resúmenes de Interfaces 
7 en Palermo. Congreso para Docentes, Directivos, Profesionales e Instituciones de Nivel Medio y Superior. CABA, 21 de mayo de 2019. Año XIV, Vol. 42, Mayo 2019. Disponible en: https://fido.palermo.edu/servicios_dyc/publicacionesdc/ archivos/766_libro.pdf

2019. Disponible en: https://fido.palermo.edu/servicios_dyc/publicacionesdc/ archivos/766_libro.pdf 